\title{
THE HERACLITEAN NATURE OF TIME IN THE PLATFORM FOR LIFE AND ITS RELATIONSHIP WITH PARMENIDEAN REALITY
}

\author{
R.G. BOOTHROYD \\ Chartered Engineer, Queensland, Australia.
}

\begin{abstract}
Any phenomenon which can artificially change the rate at which time passes must describe natural statistical variations truly and to the altered time-scale. These necessary criteria are specified here in terms of correlation coefficients. The strange nature of Heraclitean time is examined further. Accepting the limited present scientific knowledge available, it seems reasonable that quantum decoherence can explain the development of Heraclitean time from Parmenidean true reality with regard to present and future time. However, past-time is far more mysterious and alternative possible suggestions are considered. Experimental clarification of these would be expensive and difficult. This depends on our ability to extend the coherence time of the wave function in Schrödinger's equation in the present instant. It seems that this is the only feature of time available to us for experimental examination. It also seems that the best way to achieve experimental success is to exploit present-day developments in qubit design in quantum computer research which faces the same basic technical problem.

Keywords: Darwinian evolution, Heraclitean time, Parmenidean time, quantum decoherence, quantum entanglement, qubit design.
\end{abstract}

\section{INTRODUCTION}

In an earlier paper [1] one strange observed conclusion was that the equations derived were applicable from both a Heraclitean and also a Parmenidean viewpoint. For an explanation of these viewpoints see Appendix I.

Equation (5) in reference [1] must be the most simple of all equations in space-time but it does include all 4 necessary dimensions (circular pipe diameter is 2 dimensions and flow in a fully-developed turbulent boundary layer eliminates the relevance of the third axial dimension).

Using fluid dynamics it was shown that time can move faster or slower in different models yet preserving time's statistical nature. This is the only example of true controllable time variation known to the present author but this does not preclude the possibility of other situations showing this same effect. However such other phenomena would also need to satisfy certain criteria which are detailed in section (2).

\section{CRITERIA FOR MODELLING TIME-DEPENDANT PHENOMENA}

In the previous paper [1], it was shown that time effects can be modelled truly to a different rate of exposure of sequential events but otherwise the contracted or stretched time differed in no other way from the time in which we live.

It is also a reasonable supposition that, within reasonable limits, life forms could develop in such different time systems according to the principles of Darwinian evolution. 
However any platform for life, as we know it, needs an unchanging forward arrow of time, preferably with reliability of event sequencing. In particular, clearly it is essential that the progression of events must be permanent and unstoppable by any physical natural effect. It also seems reasonable to suspect that Heraclitean time may be triggered by an extremely strong and repetitive instability effect followed by a transition to a very stable 'ground state'. This control system is expected to be a fundamental property of Nature which cannot be negated by any other natural interfering activity. This conclusion arises from the observation that the nature of time in which we live does not appear to change in any way. Quantum decoherence is thus likely to be an explanation for this important control feature in Nature. At this stage, our understanding of the physical mechanism of quantum decoherence is insufficient to pursue this investigation much further [2].

Any other physical phenomenon, which we might observe, in which time events appear to be sequenced differently, needs to be checked and evaluated to discriminate it from simple data-logging devices and other equipment which merely record data. Such devices do not simulate time because, as mere copying machines, they are incapable of modelling the statistically varying nature of events in time.

\subsection{Modelling of statistical phenomena: testing details}

The earlier example [1] serves to demonstrate the necessary considerations just mentioned. The following tests can be adapted easily to other statistically-based phenomena as the method uses standard Pearson correlation coefficients. Expressing Pearson correlation coefficients mathematically may seem rather ponderous but in practice their use is little more than applying common sense.

Confirmation that the statistical nature of time is being modelled correctly is best clarified by studying related event-detail which is described for turbulent fluid motion in expressions such as:

$$
\mathrm{R}=\mathrm{u}_{1}{ }^{\prime} \mathrm{v}_{1}{ }^{\prime} / \sqrt{ } \mathrm{u}_{1}{ }^{2} \cdot \sqrt{ } \mathrm{v}_{1}{ }^{\prime 2}
$$

Here, for greater clarity of explanation we use, 3-dimensional rectangular Cartesian coordinates of space, $\mathrm{x}, \mathrm{y}, \mathrm{z}$, and corresponding fluid velocities $\mathrm{u}, \mathrm{v}$, and $\mathrm{w}$ instead of polar-cylindrical coordinates. Thus:

$$
\mathrm{u}=\mathrm{U}+\mathrm{u}^{\prime}(\mathrm{t}) ; \mathrm{v}=\mathrm{V}+\mathrm{v}^{\prime}(\mathrm{t}) ; \mathrm{w}=\mathrm{W}+\mathrm{w}^{\prime}(\mathrm{t})
$$

and eqn (1) is a detail of a single instantaneous event.

$\mathrm{U}, \mathrm{V}, \mathrm{W}$ are the time (t) averaged constant velocities and the lower case values are all time-varying. Various configurations of eqn (1) can be used and an example, similar to all of these, is detailed in Appendix II.

In any truly modelled state such as in reference [1] and using this paper's nomenclature, $R_{d}$ for system A would not correlate with $\mathrm{R}_{\mathrm{d}}$ for system $\mathrm{B}$. On the other hand, for a data logger, if $R_{d}$ is scanned over a period of time, there would normally be instances where intermittent regularly spaced spikes of correlation would be seen, i.e.:

$\mathrm{R}_{\mathrm{dA}}=\mathrm{R}_{\mathrm{dB}}$ for short intervals of time but this equality is not observed in true statistical modelling. This shows the simple copying effect of a data-logger.

However, in testing a true modelling situation of systems A and B, again in the nomenclature of [1] and Appendix II in the present paper, we need to average the statistics over a period of time. Correct statistical averaging should then give :

$$
\mathrm{R}_{\theta, \mathrm{d}^{*}, \mathrm{~A}}=\mathrm{R}_{\theta, \mathrm{d} * \mathrm{~B}} \text { where: }
$$




$$
\theta_{\mathrm{A}} / \theta_{\mathrm{B}}=\mathrm{T}_{\mathrm{A}} / \mathrm{T}_{\mathrm{B}}=\left(\mathrm{D}_{\mathrm{A}} / \mathrm{D}_{\mathrm{B}}\right)^{2}
$$

The latter equality is only for the simple case of 2 circular pipes $A$ and $B$ with diameters $\mathrm{D}_{\mathrm{A}}$ and $\mathrm{D}_{\mathrm{B}}[1]$.

\section{NATURE OF TIME}

Figure 1 illustrates the way in which time in our world seems to originate. Figure 1 is also intended to illustrate the basic model to be used for future investigation. A static diagram in Fig. 1 has its limitations pictorially. For example, it is conceivable that events can move either to the past or the future but both would be expected to be invisible to us in this case. The various arrows in Fig. 1 indicate information transfer and physical-change processes. The wavy enclosure (E) is the world in which we live: it is the world of Heraclitean time. Real time (P in Fig. 1) is Parmenidean in nature and exists outside of our experience. Also it has long been known that there is no such thing as moving time, as we know it, in Parmenidean existence. It is surmised that there are only events.

It seems almost impossible to investigate $\mathrm{P}$ by today's scientific methods but philosophical/theological reasoning appears to present plausible truth (see end of section (5)) to help us to make progress. Also, there are plausible observations described in sections (5) and (6) that $\mathrm{P}$ and $\mathrm{E}$ may sometimes both exhibit themselves together in close proximity to a limited degree in our own world where we exist in Heraclitean time. The established literature [3-5] casts no reasonable doubt on the suggestion that Heraclitean time originates from Parmenidean existence through the processes described relating to item A in Fig. 1. A is the phenomenon of quantum decoherence, sometimes described as universal entanglement [3]. The actual physical mechanism of wave-function collapse in decoherence is not understood yet. However, because time exists everywhere in our known universe, it seems to be related to physical matter in some way as yet unknown. Penrose has been more specific in attributing wave-function collapse to gravity [2].

\subsection{Experiments with a 'toy' universe}

Although theoretical studies have long revealed that time does not have a real flowing nature, only recently has this been clarified experimentally [6]. As is the case in most papers on quantum mechanics, reference [6] is written in Dirac (a.k.a. bra-ket) mathematical

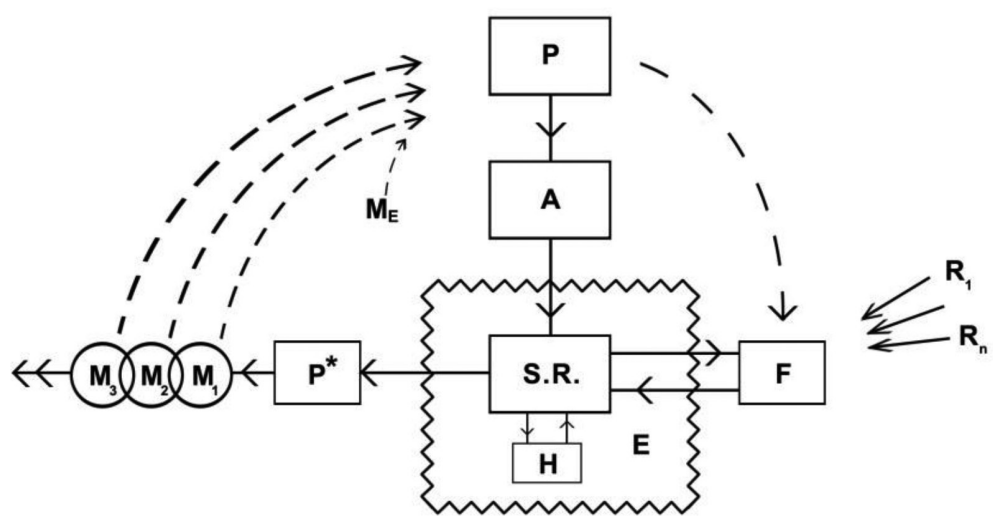

Figure 1: Suggested mode of generation of Heraclitean time. 
notation [7, p. 254-258]. Reference [8], because it does not use bra-ket notation, is particularly useful in explaining reference [6] and the earlier theoretical history preceding it.

It will be apparent in section (6) that the smallest particles of matter are the least affected by environmental decoherence [2]. In reference [6] two photons were used in an entangled manner. One of them was used as a form of primitive clock to evaluate the evolution of the other. The observed photon was evaluated externally where it exhibited no apparent change with time. It was also demonstrated that time changed as we know it when the evolving photon was examined internally, i.e. within the system. The evolution of the photons was produced by changing their polarisation. This was done by passing them through birefringent quartz plates of different thickness.

The experiment demonstrated that time appears to be an emergent property of quantum physics but only when it is observed from inside this 'toy' universe. However, this experiment does not explain the actual physical mechanism of sequential and ordered time generation from the entanglement process which we always observe in our platform for life.

\subsection{Present time}

Without the phenomenon of time, which appears to us to flow sequentially in a most ordered way, all life on our planet could not even exist. Life depends on chemistry and all chemical reactions develop over a period of time. Also 'cause' must always precede any 'effect' (principle of causality). Additionally, all life forms evolve according to Darwinian principles of evolution and this requires constantly flowing time and causality [9]. Major excursion events (important in the development of new biological species) in evolution [10] also rely on time and also on its statistical nature. Yet in reality (section 3.1) it has long been known that time does not flow at all, even though it appears to be the case in our world.

The forward arrow of time is apparent in the whole universe known to us and involves thermodynamic irreversibility and nett entropy increase. All 3 of these factors have a strangeness which makes them unique compared with the time reversal invariance of most equations which describe fundamental physics. The inevitability of death/decay and perpetual renewal by rebirth is universal in Nature. Our world and its environment is enormous compared with the tiny 'toy' universe examined [6] yet their underlying physical phenomena appear to be similar.

The strange features of life on our planet are usually considered scientifically to be mere freaks of nature, but the more we learn, the more this argument seems to be losing ground to the suspicion that Nature is contrived by an external mechanism. This view can be supported scientifically [11-13] and remains of interest [14], even though 'life' is shrouded in statistical uncertainty which may seem to give considerable credence to the former interpretation of many "freaks in Nature". Nevertheless, it remains reasonable to conclude that we cannot dismiss the concept of intelligent design in Nature.

This paper attempts to throw some light on these issues. Even so, inevitably, it asks far more questions than it can answer. It entails reasonable suspicions which are not yet hard facts and it is explorative rather than factual in its approach. Our strange world cannot escape this continued scientific scrutiny and comment in today's rational society.

\subsection{Future time}

Although mankind (and to a lesser extent many other animal species) is conscious of future (and past) events, Heraclitean time restricts observation of these events to the present instant 
only. The reasons for this, if any, are uncertain, yet explainable to some extent. Yet if, as is supposed here, Heraclitean time derives from Parmenidean reality, then it is reasonable enough to expect this.

The human brain has been well studied [15] and shown to have adapted to using reasoning to make interpretations from this sequence of observed events exhibited by Heraclitean time. Visual data, in particular, have been shown to be sorted by the brain to give us an adequate sense of our environment. Insufficient data are 'fleshed out' i.e. integrated when necessary and surplus data are rejected at other times to optimise human reaction to circumstances.

The future is always perceived in the present instant. This is an inevitable consequence of quantum decoherence which appears to be the universal physical mechanism at work to offer us a single reality at the instant of observation when all other wave-functions have collapsed.

Yet higher forms of animal life, especially ourselves, have the ability to plan and change the future. Future events, for us, are composed of a planned component and a statistically uncertain component. The latter is where it is beyond our ability to predict future events in their entirety. It even seems likely that the higher the scientific level of a humanoid civilisation, the higher is the likely planned component of future events.

Climate control is an ideal example of our ability to control the future. Global warming can be avoided by declining to use fossil fuels, but the consequences are uncertain. There is even the danger of overcompensation which might, or might not, occur from statistical uncertainty in the future. This might take the form of an unexpected surge in volcanic activity reducing Earth's absorbed solar radiation by atmospheric dust contamination. Another cause might be from an extensive war with nuclear weapons which could stimulate a 'nuclear winter'. These comments are not intended to discourage the use of alternatives to fossil fuels but to emphasise that our future planning is always uncertain.

Automatic control systems may appear to control much of our future today. However, they can only function when subject to quantum effects in the present instant because quantum decoherence always makes any control action a present event. As might be expected, closed loop quantum control systems which are designed to be linear, and have continuous feedback, normally become non-linear systems in practice [16].

Thus effectively it is reasonable to conclude that the future is merely a succession of present events which will reach us in due course. These random effects are shown as $R_{1}-R_{n}$ in Fig. 1. They are a part of our world but they are outside the present instant of time.

\subsection{Past time}

Past time is altogether more mysterious. To start with, it is important to emphasise that we have no means by which to examine the past directly. We can examine the effects of aging but such records ( $\mathrm{H}$ in Fig. 1) are only 'present instances' of a recording made which can be examined at another later 'present instant'. Such records have nothing at all to do with a true past. It is concluded that past records of a present instant are not evidence that a true past time even exists.

Yet we cannot dismiss easily the possibility that what we presume to be the past may even be able to develop an existence of its own. Prigogine's entropic barrier [1, pp. 203-205] alone explains why we cannot see any such past existence ( $\mathrm{P}^{*}$ in Fig. 1). Figure 1 also shows a consecutive series of presumed individual pasts $\left(M_{1}-M_{n}\right)$ as they recede and become increasingly inaccessible, due to entropy, from our present instant of time. Yet these 'M- type' hypothetical existences may be able to transfer information with each other, even if they still exist in Heraclitian time, because the entropy barrier transfer problem is minimal for these 
adjacent $\mathrm{M}$ systems. This effect is shown by overlapping the circles in Fig. 1. In examining this hypothetical situation, there is a significant difference between inanimate and living objects because of the differing levels and mode of entropy transfer required. It is concluded that we do not know anything about the true past, or even if the past may itself even revert to a Parmenidean state, also shown by dotted lines in Fig. 1.

However, with some considerable difficulty, it may be possible to investigate the nature of past time experimentally. This is discussed in section (6)

The above description of a presumed past time bears a resemblance to a more limited form of the multiverse theory first proposed by Everett [17]. Although controversial, the multiverse theory is now under serious consideration by a large number of scientists $[18,19$, pp. $205-$ 208]. The multiple universe concept is shown as $M_{E}$ in Fig. 1. It is difficult to know where to fit $\mathrm{M}_{\mathrm{E}}$ in Fig. 1. Multiple universes are alleged to develop before decoherence (A in Fig.1). There is no known reason for them to revert to the Parmenidean state as shown in Fig. 1 but, if they exist, they are likely to be similar in being complex Hilbert space systems.

The mechanism of generation of Everett's multiverses is quite different from the mechanisms considered in this paper. Multiverses are hypothetical parallel universes existing outside the decoherence effect, whereas those considered as $M_{1}-M_{n}$ in Fig. 1 are 'in series', that is consecutive forms of suggested possible existence which have already experienced quantum decoherence.

The problem with multiverse theory is that it cannot be examined experimentally. However, as discussed in section (6), although it is difficult, examination of the model of past time in the present paper may be amenable to experimental investigation. If such true past existence can be demonstrated, there is also a reasonable possibility that such a separate existence may even be able to move backwards or forwards in time as we know it.

\section{SPECIAL REALITY AND TRUE REALITY}

The world in which we live is called 'Special Reality' in the present paper. This is shown as S.R. in the wavy enclosure E in Fig. 1.

Much of 'Special Reality' presents complicating issues such as the forward arrow of time, irreversibility and entropy. In addition so many other factors in modern physics such as accounting for dark matter and dark energy are now being consigned to the 'too hard basket', even though they need explanation in a comprehensive account of existence in Special Reality. Perhaps a better understanding of the true physical nature of quantum phenomena would help us to understand our world more clearly? Perhaps we should follow the example of Einstein himself and sort out physical reality first and then try to cope with the mathematics later? Today we have better computers and improved numerical methods if the mathematics gets too difficult. In an earlier age Einstein managed, after a long struggle, to finalise his General Theory of Relativity without the superior facilities we now have at our disposal.

\section{SCHRÖDINGER'S EQUATION AND THE SUSPECTED HERACLITEAN/ PARMENIDEAN INTERFACE}

The existence of matter waves was proposed by de Broglie in his Ph.D. thesis. These waves are normally considered as a mathematical abstraction. They are unlike any other wave phenomenon in our experience. Large objects in our world are associated with matter waves with wave-lengths that are too small to be significant. The matter wavelength $(\lambda)$ is given by:

$$
\lambda=\mathrm{h} / \mathrm{p}
$$


where $\mathrm{p}$ is the momentum of the matter considered and $\mathrm{h}$ is Planck's constant. Usually only by cooling a small particle to near absolute zero does the wavelength become large enough to be of importance.

Also, it is apparent from eqn (2), that only for very small particles such as an electron is the de Broglie wavelength relevant. If we adopt the typical professional engineer's attitude that equations often have a limited range of relevance and application, then we can ignore matter waves in much of our everyday world. We only have to consider matter waves for the smallest elements of matter. Yet it is these small elements of matter which seem to exercise considerable control over our existence. Quantum control appears to be a real effect.

Schrödinger formalised de Broglie's concept into his well-known equation:

$$
-h^{2} / 2 \mathrm{~m} .\left\{\nabla^{2} \psi(\mathbf{r}, \mathrm{t})\right\}+\nabla \Psi(\mathbf{r}, \mathrm{t})=\mathrm{ih} \partial \Psi(\mathbf{r}, \mathrm{t}) / \partial \mathrm{t}
$$

where $\mathbf{r}$ is the position vector and $\mathrm{h}$ is Planck's constant divided by $2 \pi$

The first term describes the movement of this wave function $\Psi$, the second term describes the consequences on $\Psi$ of forces acting on the particle and the third term on the right hand side of the equation describes how the wave function changes with time as we know it. $i=(-1)^{1 / 2}$ is especially significant in this third term producing complex numbers in any solution to eqn (3). Schrödinger's equation has the advantage of being linear and normally is composed of a number of superposed solutions. It is also of interest that it is time reversal invariant.

Solution of eqn (3) for $\psi(\mathrm{t})$ allows the spatial movement of a small particle to be predicted statistically thus allowing Heraclitian time to be related to $\Psi(\mathrm{t})$. It seems reasonable to suppose that the wavefunction $\Psi(\mathrm{t})$ derives initially from the Parmenidian state as it cannot survive at all in the Heraclitean state where it is swamped by other wave functions emanating continuously from adjacent matter comprising many other atoms. It is considered that this feature is the one which makes eqn (3) so useful as it appears to make it a natural bridge between the Parmenidian and Heraclitean states. The latter is a 4 dimensional existence in space-time with which we are all familiar as we live in it. The Parmenidian state is almost certainly one which includes other 'Hilbert' space dimensions which would explain why it is invisible and beyond our scrutiny. This same problem is also encountered in string theory [19, pp. 327-375] and the subsequently-developed M (membrane) theory [19, 376-412]. Both of these widely investigated theories also cannot be investigated experimentally for much the same reason.

As we lack a scientific method of interpreting the Parmenidean state which we cannot see, it seems that the best we can do is to adopt a very rational metaphysical argument [20 pp. 142-146]. If this approach (see Appendix III) proves to be valid, it would also provide us with an explanation for the puzzling instantaneous communication between quantum-entangled matter when it is, presumably, in the Parmenidean state and quantum non-locality applies [21]. It would also support our fundamental belief in the lack of sequentially moving time as we know it unless it is part of this strange and abnormal Heraclitean state which is called 'special reality' in the present paper to distinguish it from the 'true reality' of the Parmenidean state.

\section{EXPERIMENTAL PROBLEMS AND A SUGGESTED SOLUTION}

It appears that the only option available to us for examining the nature of time experimentally is to investigate the present instant. Prima facie this suggests that it is extremely difficult to study the features in Fig.1 in this way. If we are to make progress in investigating the nature of time in Special Reality, then we must learn how to alter quantum decoherence so as to understand its mechanism. We are, by now becoming sufficiently aware that decoherence is 
one of the most powerful control systems in our Heraclitean world and that, in part, it seems to account for the very surprising stability of the material world in which we live.

Good fortune seems to have come to the aid of our curiosity. This is the present worldwide effort in developing a quantum computer. The overriding main problem in designing a quantum computer also lies with the decoherence problem, the very same issue involved in studying the features of time itself, as depicted in Fig. 1. A vast amount of present day research effort is directed at looking at different qubit designs in which decoherence can be retarded sufficiently for a quantum computer to carry out calculations without error. It seems that the main interest in quantum computers is a military one. If a mere 1000 or so qubits can work together without one of them suffering decoherence to cause machine failure, then this powerful computer system would be expected to be able to decode the most carefully encrypted message while it is actually being transmitted. Such a device would be an awesome weapon in military intelligence. Military projects are well-funded and are often successful as a result. Spin-off, incorporating socially-useful technology, is often a bonus from such military research.

Exploiting this quantum computer research seems to be the path to take in trying to understand the physics of time. There are other major projects relevant to improving our understanding of the physics of time. Research at CERN near Geneva has delivered a very comprehensive account of the subatomic nature of matter. Yet much further study is required in understanding the meaning of this wealth of data. Understanding the presumed influence of matter on quantum decoherence also appears to be essential to understanding the true nature of necessary stability in our platform for life.

It is premature to decide how best to use this research. One obvious approach is to use a quantum computer to work out a mathematical procedure of special design and examine the adverse influence on the calculation procedure as decoherence causes the quantum computer to malfunction. Even more interesting is the possibility of exploring the changes $M_{1}-M_{n}$ in Fig.1. Examining possible interaction, using 2 small (less than 20 qubit) computers in different configurations in developing a mathematical calculation, may be of much interest. This may be able throw light on the mysterious physical nature of time-past.

A few brief words about qubit design seem relevant. Whereas the best approach in designing a qubit has not yet been established, some general conclusions are important. The smaller the amount of matter in a qubit, the easier it is to isolate it from the environment which is the cause of decoherence. For this reason qubits therefore often use ions or electrons, as their working component. An extremely high vacuum surrounds the small amount of matter used and temperatures close to absolute zero delay the onset of decoherence. The quantum Zeno effect [22] can also delay wave-function breakdown. Magnetic fields have relatively little decoherence effect and are useful in gate design to transfer information within the computer as well as delaying the onset of wave-function collapse. Larger qubits such as Josephson junctions also are of interest. These use a superconducting circuit interrupted by a very thin insulating film allowing the quantum tunnelling effect. Interestingly, this is where there is close proximity of matter where thermodynamic reversibility and highly irreversible processes occur close together. However, to the best of the present writer's knowledge no significance has yet been attached to this factor.

\section{PHYSICS AND NATURE IN HERACLITIA AND PARMENIDIA}

Because there is no such thing as time in a Parmenidean world, it seems to be very different from our own world, so let us, for the want of a name, call this other existence Parmenidia. It seems to be a world of instants but they do not appear to be sequenced as is the case in our 
world. The absence of 'time' as we know it, also implies that there is no place for irreversibility, and hence there would be no entropy. The nature, and even the existence, of matter/ energy in Parmenidia seems to be a matter of conjecture at this stage.

The absence of Heraclitian time seems to have its disadvantages in Parmenidia. One of these is that there is no apparent mechanism for the development of life. Nevertheless speculation about the existence of life is, like matter and energy, a subject of conjecture. Nevertheless, it is reasonable to suspect that Parmenidia is likely to be a sterile place. Our own experience of life is limited to life based on chemical process which requires Heraclitean time.

Yet Parmenidia seems to be a place with much less restrictions when compared to our own world, which for the sake of comparison, we will call Heraclitia. In Parmenidia there is no reason to suspect any restriction on viewing instants [20] and communication seems to be without the restrictions which we experience in Heraclitia where no form of communication can exceed the velocity of light.

If Parmenidia is the true reality, then its world of physics seems to be much more simple than in the world of Heraclitia where irreversibility, time and entropy complicate our physics. Perhaps our research in physics would become much clearer if we could, at some future date, learn how to examine it from the Parmenidean viewpoint?

\section{DISCUSSION INCLUDING THEOLOGICAL CONSIDERATIONS}

Modern science is slowly unravelling the physics of Nature's natural control systems. Their complexity and sophistication are astonishing. Climate control [23] has a multitude of both negative and positive feedback systems which act together effectively. The same can be said of predator/prey dynamics and dynamic population control of species. All these mechanisms are statistical in nature and could indeed be regarded as mere freaks of nature.

Predator/prey dynamics is so offensive to human sensitivity that it is hard to conceive of a reputedly benign creator god designing such an objectionable system. Yet a 'true believer' scientist could counter this assertion with a challenge to design a more reliable system of species population control which was just as efficient, automatic, self-adjusting and reliable. A theologian [24, p. 98] might well support the scientist, pointing out the importance of ensuring that the ways of God should remain hidden, perhaps even secret, but at least unobtrusive. If this was not the case, the human species would lose its freedom of choice to develop naturally and we would be more like slaves or machines.

This example is quoted to demonstrate the apparent futility of trying to use science to prove the existence or non-existence of God. But for those of us who choose to want to believe in God, science can be an aid to demonstrate rational purpose to support our belief.

Sometimes our theological reasoning can 'run off the rails', often with delusions of self-importance concerning our species. It is unlikely that we are the only highly-developed, human-like species possessing deep religious insight. The diagram on page 42 of reference [25] shows that, as at 2014, we had identified 1771 planets of which only 14 could support life as we know it. Two of the 14 were Earth and Mars. Actually 14 out of 1771 is quite a high proportion. Even the most simplistic calculation concerning the known size of the Universe suggests that a presumed Creator-God who uses statistically-based methods seems likely to have 'hedged bets' with regard to 'Goldilocks Planets' like our own.

The idea of 'betting' often seems distasteful to the scientific mind. Much has been written about Albert Einstein's outburst declaring that "God does not play dice". Did the realisation that Nature is statistical flash across his mind? We can never invade the inner privacy of anyone's mind and the thoughts which crossed the great man's mind are lost to us for ever. 
Perhaps the outburst was just a feeling of distress that our very human desire for orderliness and understanding is so often subordinate to the vagaries of the statistical component in Nature [26].

\section{CONCLUSIONS}

When we consider the needs of all life-forms on our planet, it is essential that we have a forward-moving irreversible arrow of time. Yet this is contrary to the general mathematical nature of physical laws in the Cosmos. Preferably, this necessary form of time should move at a constant rate but it must certainly never be made to stop. This is the situation we observe. As long as it is within reasonable limits, the rate at which time evolves [1] does not seem to be an important factor as life would be able to adapt to it. However the fact that time in real reality (called the state of Parmenidia/True Reality in the present paper) does not have these necessary properties suggests that our platform for life (called Special Reality/ Heraclitia in this paper) is changed by an exceptionally reliable control system. We know this as quantum decoherence which can give us the basic requirements for our existence. This quantum effect is one of the most powerful, universal, speedy, effective and reliable effects in Nature.

Special Reality, where we live and see, seems to derive from True Reality, where we cannot live and cannot see. Quantum decoherence, sometimes called universal entanglement, is almost but not quite instantaneous. Only for the smallest items of matter can decoherence be delayed sufficiently for experimental investigation. Fortunately the design of workable qubits for future quantum computers faces exactly the same problem of controlling decoherence. Consequently, it makes sense to try to use this research activity to help elucidate the nature of Heraclitean time as a useful side-line of general research.

Special Reality seems to involve many complications compared with True Reality and it is hoped that this proposed line of investigation may also eventually help us to clarify some of today's outstanding questions in modern physics.

\section{REFERENCES}

[1] Boothroyd, R.G., Non-relativistic time, existence and adaptation. International Journal of Design and Nature and Ecodynamics, 10(3), pp. 193-212, 2015. http://dx.doi.org/10.2495/DNE-V10-N3-199-212

[2] Brooks, M., The secret life of reality. New Scientist, 205(3002), pp. 26-29, 2015. http://dx.doi.org/10.1016/S0262-4079(15)60031-8

[3] Decoherence website, available at: www.decoherence.de/home.html (retrieved 2/12/2015) 2015.

[4] Idem. ibid. Zeh, H.D., Three essays: a) the essence of the concept of decoherence, b) how decoherence can solve the measurement problem, c) quantum nonlocality and Einstein locality.

[5] Idem ibid. Joos, E., Essay on; 'do we need observables?'.

[6] Moreva, A., Brida, G., Gramegna, M., Giovannetti, V., Maccone, L. \& Genovese, M., Time from quantum entanglement: an experimental illustration. Physical Review, A89, 052122, 2014. http://dx.doi.org/10.1103/PhysRevA.89.052122

[7] Bub, J., Interpreting the Quantum World, Cambridge University Press, pp. 1-290, 1997.

[8] Anon. Quantum experiment shows how time 'emerges' from entanglement, 2015, available at: https.//medium.com/the-phtsics-arxiv-blog/quantum-experiment shows how time-em 
[9] Hawks, J., Still evolving after all these years. Scientific American, 31(3), pp. 70-72, 2014. http://dx.doi.org/10.1038/scientificamerican0914-86

[10] Dewel, G., Kondepudi, D. \& Prigogine, I., Chemistry far from equilibrium: thermodynamics, order and chaos. In The New Chemistry, ed. N. Hall, Cambridge University Press, pp. 440-464, 2000.

[11] Rees, M.R., Just Six Numbers, Weidenfeld \& Nicholson: London, pp. 1-194, 1999.

[12] Denton, M.J., Nature's Destiny, Simon \& Schuster: New York, pp. 1-454, 1998.

[13] Wilczek, F., The Lightness of Being: Big Questions, Real Answers, Penguin: London, pp. 1-270, 2008.

[14] Slezak, M., Was the Universe made for us. New Scientist, 225 (3019), p. 32, 2015. http://dx.doi.org/10.1016/s0262-4079(15)30260-8

[15] Spinney, L., Once upon a time. New Scientist, 225(3003), pp. 28-31, 2015. http://dx.doi.org/10.1016/S0262-4079(15)60075-6

[16] Jacobs, K., Application of feedback control in quantum systems. ArXiv:quantph/0605015 v1, p. 8, 2nd May, 2006.

[17] Hooper, R., Life in the multiverse. New Scientist, 223(2988), pp. 32-37, 2014. http://dx.doi.org/10.1016/S0262-4079(14)61861-3

[18] Deutsch, D., The Fabric of Reality, Penguin: London, pp. 190-193, 1998.

[19] Greene, B., The of Fabric of the Cosmos, Penguin: London, pp. 1-569, 2004.

[20] Lewis, C.S., Time and beyond time. In Mere Christianity, HarperCollins: Glasgow, U.K., pp. 142-146, 1984.

[21] Popescu, S., Non-locality beyond quantum mechanics. Nature Physics, 10, pp. 264 270, 2014.

http://dx.doi.org/10.1038/nphys2916

[22] Itano, W.M., Perspectives on the quantum Zeno paradox. Journal of Physics: Conference Series 196, 012018, 2009. http://dx.doi.org/10.1088/1742-6596/196/1/012018

[23] Hansen, J., Storms of my Grandchildren, Bloomsbury: New York, pp. 6-58, 70-89, 2009.

[24] Vanier, J., Life's Great Questions, Franciscan media: Cincinnati, USA, pp. 1-172, 2015.

[25] Lemonick, M.D., The hunt for life beyond earth. National Geographic, 226(1), pp. 26-45, 2014.

[26] Bohm, D., A suggested interpretation of the quantum theory in terms of hidden variables I. Physical Review, 85(2), pp. 166-179, 1952.

http://dx.doi.org/10.1103/PhysRev.85.166

\section{APPENDIX I: AN EXCEEDINGLY BRIEF HISTORY OF TIME}

(N.B. References which apply only to this Appendix are prefixed with 'A' and are listed at the end of this Appendix.)

Heraclitus and Parmenides were two famous Greek philosophers who lived in about 500 BCE. Heraclitus, who believed that 'everything flows', including time, differed from Parmenides who thought that 'all existence is timeless'. With regard to time, modern science has concluded that Parmenides was right and Heraclitus was wrong. Yet to those of us who tend to rely on common sense rather than scientific reason, it seems that it was Heraclitus who appeared to be right and Parmenides was wrong. However, it is now beyond dispute that Parmenides was right and time does not flow, even if it seems to behave as if it had this 
property. Today we know that time is just a series of present events (moments), often of short duration, sequencing in one direction only towards increasing age. The number of such sequences equates to the number of observations made by a human observer.

We have no means by which to alter the passage of time in which we live. Perhaps this has led us to some incorrect conclusions [A1, pp. 254-258] that time is merely an illusion in the human mind. The present paper takes an opposite view and describes the time in which we live as 'Special Reality' but it also refers to an equally important 'True Reality'.

Many eminent scientists have spent at least a little of their lives examining problems associated with time. Some of these have written excellent books for the general public which use the very minimum of mathematics. The most famous of these texts is that of Stephen Hawking [A2]. Another excellent text is that of Brian Greene referred to in this paper [19]. Both of these leading scientists also often appear on television in well-presented documentaries, sometimes talking about the physics of time.

However, for members of the general public who wish to read extensively in order to gain a better understanding of the nature of time, the books of another eminent theoretical physicist, Paul Davies, are particularly recommended. Davies wrote about 20 of these books in inexpensive paperback form and he covers a huge area of relevant physics in very readable detail. The following examples are especially useful [A1, A3-A6].

For readers with more limited time at their disposal, a less well known paperback [A7], covers all aspects of the subject of time. It contains humorous cartoons on every page depicting human perplexity as we puzzle over the complexity of our existence.

[A1] Davies, P., About Time, Penguin: London, pp. 1-316, 1995.

[A2] Hawking, S., A Brief History of Time, Bantam: New York, editions 1996, 1998, 2005, (the first, 1988 edition, seems to have become an expensive collector's item. Later editions contain more material.

[A3] Davies, P., God and the New Physics, Simon \& Schuster: New York, pp. 1-255, 1983.

[A4] Davies, P., The Cosmic Blueprint, Simon \& Schuster: New York, pp. 1-222, 1988.

[A5] Davies, P., The Goldilocks Enigma, Houghton Mifflin: Boston, pp. 1-315, 2006.

[A6] Davies, P., The Mind of God, Penguin: London, pp.1-254, 1992.

[A7] Callender, C. \& Edney, R., Introducing Time, Icon Books: UK. pp. 1-114, 2004.

\section{APPENDIX II: NOMENCLATURE FOR SECTION (2) AND EXAMPLE OF CORRELATION COEFFICIENT}

Event eqn (1) gives us a value of $\mathrm{R}$ in the range $0<\mathrm{R}<1$, where the extrema represent zero or full correlation of $\mathrm{u}^{\prime}$ and $\mathrm{v}^{\prime}$. The Eulerian coordinate system is used because turbulence measurements are normally made this way. If turbulent dispersion measurements using a tracer were made as an alternative, the Lagrangian coordinate system (moving with a fluid element) would also yield an acceptable but similar alternative analysis. In this case the functional dependence $(\mathbf{r}+\mathbf{d})$ in eqn (A1) is replaced by a variable time interval.

A typical instantaneous correlation coefficient for two measurements a distance $\mathbf{d}$ apart is obtained by averaging over time a typical event-log for $\mathrm{R}_{\mathrm{d}}$ such as:

$$
\mathrm{R}_{\mathrm{d}}=\mathrm{u}^{\prime}(\mathbf{r}) \mathrm{v}^{\prime}(\mathbf{r}+\mathbf{d}) /\left\{\left(\mathrm{u}^{\prime}\right)^{2}\right\}^{1 / 2} \cdot\left\{\left(\mathrm{v}^{\prime}\right)^{2}\right\}^{1 / 2}
$$

where vectors are only indicated where there is a functional dependence.

This expression may be integrated over a time interval $\theta$ to yield one of many similar types of correlation coefficient. For eqn (A1) this is: 


$$
R_{d, \theta}=\frac{\int_{0}^{\theta} u^{\prime} v^{\prime}(\underset{\sim}{d}) d \theta}{\sqrt{\int_{0}^{\theta} u^{\prime 2} d \theta \cdot \sqrt{\int_{0}^{\theta} v^{\prime 2}}(\underset{\sim}{d}) d \theta}}
$$

using the coordinate origin where $\mathrm{u}^{\prime}$ is measured and noting the convergence of the value of $\mathrm{R}_{\mathrm{d}, \theta}$ as $\theta$ increases. It is also necessary to take account of $\theta$ according to the time-scale relation: $\theta_{\mathrm{A}} / \theta_{\mathrm{B}}=\mathrm{T}_{\mathrm{A}} / \mathrm{T}_{\mathrm{B}}$ in the last equation in section 2 above.

Experimental data used in eqn (A2) may be smoothed for accuracy in calculation.

The calculated value of eqn (A2) is repeated for any other simulation of time by scaling the appropriate values of $\mathbf{r}$ and $\mathbf{r}+\mathbf{d}$ to ensure that there is geometrical similarity of the comparison for the two systems A and B thus, using the non-dimensionising star (*) indicator in [1]:

$$
\mathbf{r}_{\mathrm{B}}^{*}=\mathbf{r}_{\mathrm{A}}^{*} \mathrm{D}_{\mathrm{B}} / \mathrm{D}_{\mathrm{A}} ; \mathbf{d}_{\mathrm{B}}^{*}=\mathbf{d}_{\mathrm{A}}{ }^{*} \mathrm{D}_{\mathrm{B}} / \mathrm{D}_{\mathrm{A}}
$$

and the two values of $\mathrm{R}_{\theta^{*}, \mathrm{~d}^{*} \mathrm{~A}}$ and $\mathrm{R}_{\theta^{*}, \mathrm{~d}{ }_{\mathrm{B}}}$ may then be compared, using eqn (A2) to check for identical correlation coefficients for systems $\mathrm{A}$ and $\mathrm{B}$.

In section (2) these correlation coefficients may involve any combination of the parameters: $\mathrm{x}, \mathrm{y}, \mathrm{z}, \mathrm{u}, \mathrm{v}$ and $\mathrm{w}$.

\section{APPENDIX III: EXCERPTS FROM REFERENCE [20]}

...A man put it to me by saying "I can believe in God all right, but what I cannot swallow is the idea of Him attending to several hundred million human beings who are all addressing Him at the same moment. And I have found that quite a lot of people feel like this."

Now the first thing to notice is that the whole sting of it comes in the words at the same moment. Most of us can imagine God attending to any number of applicants if only they came one by one and $\mathrm{He}$ had an endless time to do it in. So that what is really at the back of this difficulty is the idea of God having to fit too many things into one moment of time.

Well that is of course what happens to us. Our life comes to us moment by moment. One moment disappears before the next comes along: and there is room for very little in each. That is what Time is like. And of course you and I tend to take it for granted that this Time series - this arrangement of past, present and future - is not simply the way life comes to us but the way all things really exist.

We tend to assume that the whole universe and God himself are always moving on from past to future just as we do. But many learned men do not agree with that. It was the Theologians who first started the idea that some things are not in Time at all: later the Philosophers took it over: and now some of the scientists are doing the same.

Almost certainly God is not in Time. His life does not consist of moments following one another...

...We have to leave A before we get to B and cannot reach $\mathrm{C}$ until we leave B behind. God from above or outside of all...contains and sees it all...

All the days are 'Now' for Him. He does not remember you doing things yesterday; He simply sees you doing them, because although you have lost yesterday, He has not. He does not 'foresee' you doing things tomorrow; he simply sees you doing them...

Explanatory note added. As we are well aware, there is no restriction on our free will or any suggestion of predestination. Our future is also 'now' and visible to God. 